\title{
Dynamical Coupled-Channels Effects on Pion Photoproduction *
}

\author{
B. Juliá-Díaz, ${ }^{1,2}$ T.-S. H. Lee, ${ }^{1,3}$ A. Matsuyama, ${ }^{1,4}$ T. Sato, ${ }^{1,5}$ and L.C. Smith ${ }^{1,6}$ \\ 1 Excited Baryon Analysis Center (EBAC), \\ Thomas Jefferson National Accelerator Facility, Newport News, VA 22901, USA \\ ${ }^{2}$ Departament d'Estructura $i$ Constituents de la Matèria, \\ Universitat de Barcelona, E-08028 Barcelona, Spain \\ ${ }^{3}$ Physics Division, Argonne National Laboratory, Argonne, IL 60439, USA \\ ${ }^{4}$ Department of Physics, Shizuoka University, Shizuoka 422-8529, Japan \\ ${ }^{5}$ Department of Physics, Osaka University, Toyonaka, Osaka 560-0043, Japan \\ ${ }^{6}$ Department of Physics, University of Virginia, VA 22901, USA
}

\begin{abstract}
The electromagnetic pion production reactions are investigated within the dynamical coupledchannels model developed in Physics Reports, 439, 193 (2007). The meson-baryon channels included in this study are $\gamma N, \pi N, \eta N$, and the $\pi \Delta, \rho N$ and $\sigma N$ resonant components of the $\pi \pi N$ channel. With the hadronic parameters of the model determined in a recent study of $\pi N$ scattering, we show that the pion photoproduction data up to the second resonance region can be described to a very large extent by only adjusting the bare $\gamma N \rightarrow N^{*}$ helicity amplitudes, while the non-resonant electromagnetic couplings are taken from previous works. It is found that the coupled-channels effects can contribute about $10-20 \%$ of the production cross sections in the $\Delta$ (1232) resonance region, and can drastically change the magnitude and shape of the cross sections in the second resonance region. The importance of the off-shell effects in a dynamical approach is also demonstrated. The meson cloud effects as well as the coupled-channels contributions to the $\gamma N \rightarrow N^{*}$ form factors are found to be mainly in the low $Q^{2}$ region. For the magnetic M1 $\gamma N \rightarrow \Delta$ (1232) form factor, the results are close to that of the Sato-Lee Model. Necessary improvements to the model and future developments are discussed.
\end{abstract}

PACS numbers: 13.75.Gx, 13.60.Le, 13.60.-r, 14.20.Gk

\footnotetext{
* Notice: Authored by Jefferson Science Associates, LLC under U.S. DOE Contract No. DE-AC0506OR23177. The U.S. Government retains a non-exclusive, paid-up, irrevocable, world-wide license to publish or reproduce this manuscript for U.S. Government purposes.
} 


\section{INTRODUCTION}

It is well recognized $[1,2]$ that the data of electromagnetic meson production reactions can be used to reveal the structure of the excited states $\left(N^{*}\right)$ of the nucleon. In this paper, we report on an investigation in this direction within the dynamical coupled-channels model (MSL) presented in Ref. [3] which is being applied at the Excited Baryon Analysis Center (EBAC) of Jefferson Laboratory.

The coupled-channels approach has been used [4-7] in recent years to analyze the meson production reaction data. It is therefore useful to briefly emphasize here the essence of taking a dynamical approach as developed in Ref. [3] and in earlier works [8-19]. Since $N^{*}$ states are unstable, their structure must couple with the reaction channels in the meson production reactions. To determine correctly the spectrum of $N^{*}$ states, an analysis of the meson production data must account for the coupled-channels unitary condition. The extracted $N^{*}$ parameters can be interpreted correctly only when the reaction mechanisms in the short-range region, where we want to map out the $N^{*}$ structure, have been accounted for. The MSL model meets these two crucial requirements and is therefore suitable for analyzing the world data of meson production reactions induced by pions, photons, and electrons.

Schematically, the MSL model solves the following coupled integral equations in each partial wave

$$
T_{\alpha, \beta}\left(p_{\alpha}, p_{\beta} ; E\right)=V_{\alpha, \beta}\left(p_{\alpha}, p_{\beta}\right)+\sum_{\delta} \int p^{2} d p V_{\alpha, \delta}\left(p_{\alpha}, p\right) G_{\delta}(p, E) T_{\delta, \beta}\left(p, p_{\beta}, E\right)
$$

with

$$
V_{\alpha, \beta}\left(p_{\alpha}, p_{\beta}\right)=v_{\alpha, \beta}\left(p_{\alpha}, p_{\beta}\right)+\sum_{N^{*}} \frac{\Gamma_{N^{*}, \alpha}^{\dagger}\left(p_{\alpha}\right) \Gamma_{N^{*}, \beta}\left(p_{\beta}\right)}{E-M_{N^{*}}^{0}},
$$

where $\alpha, \beta, \delta=\gamma N, \pi N, \eta N$, and $\pi \pi N$ which has $\pi \Delta, \rho N, \sigma N$ resonant components, $G_{\delta}(p, E)$ is the propagator of channel $\delta, M_{N^{*}}^{0}$ is the mass of a bare excited nucleon state $N^{*}, v_{\alpha, \beta}$ is defined by meson-exchange mechanisms, and the $N^{*} \rightarrow \beta$ vertex interaction $\Gamma_{N^{*}, \beta}$ is related to the quark-gluon sub-structure of $N^{*}$. If we take the on-shell approximation, Eq. (1) is reduced to the following algebraic form of K-matrix models [4-6, 20-22]

$$
T_{\alpha, \beta}^{k}\left(p_{\alpha}, p_{\beta}, E\right)=\sum_{\delta} V_{\alpha, \delta}\left(p_{\alpha}, p_{\delta}\right) \times\left[\delta_{\delta, \beta}+i \rho\left(p_{\delta}\right) T_{\delta, \beta}^{k}\left(p_{\delta}, p_{\beta}, E\right)\right]
$$

where $\rho\left(p_{\delta}\right)$ is an appropriate phase space factor. Qualitatively speaking, models that make use of on-shell expressions such as Eq. (3) are used to avoid an explicit treatment of the reaction mechanisms in the short range region where we want to map out the quark-gluon sub-structure of $N^{*}$ states. Thus the $N^{*}$ parameters extracted by using Eq. (1) can be more

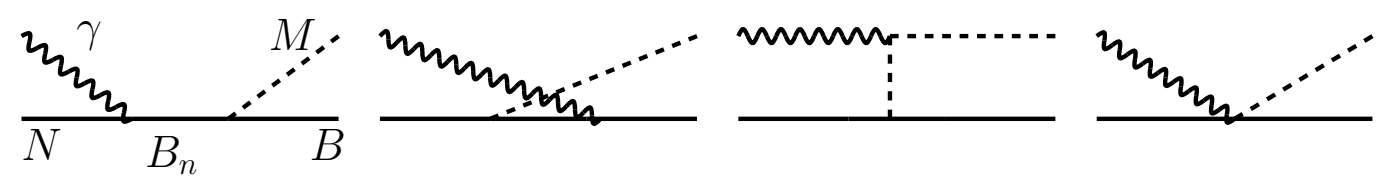

FIG. 1: The non-resonant electromagnetic meson production interaction $v_{M B, \gamma N}$, where $M B=$ $\pi N, \eta N, \pi \Delta, \rho N, \sigma N$. The details are given in Ref. [3]. 
directly interpreted in terms of the quark-gluon sub-structure of $N^{*}$. From the study $[11,19]$ in the $\Delta$ (1232) region, it is reasonable to interpret $\Gamma_{N^{*}, \beta}$ in terms of hadron structure calculations with effective degrees of freedom, such as the constituent quark model [23] and the model [24] based on Dyson-Schwinger Equations. In the near future, one hopes to relate $\Gamma_{N^{*}, \beta}$ to Lattice QCD (LQCD) calculations [25, 26]. The possibility of making contact with the current hadron structure calculations, which can be carried out with sufficient accuracy in the forseeable future, is the main motivation for performing the analysis using a technically much more involved dynamical approach.

To analyze the electromagnetic meson production reactions within the MSL model, it is necessary to first determine the hadronic part of its Hamiltonian. A progress in this direction has been made recently in Ref. [27] (JLMS). The main purpose of this work is to explore the consequence of the parameters determined by JLMS in describing the pion photoproduction reactions. Within the MSL formulation, these hadronic parameters should be consistently used to fix the strong interaction vertices of the non-resonant $\gamma N \rightarrow M B$ interaction $v_{M B, \gamma N}$ of Eq. (2). Since the electromagnetic interactions, such as the $\gamma N N, \gamma \pi \rho, \gamma \pi \omega$ vertices of the considered non-resonant mechanisms illustrated in Fig. 1, have been determined in the previous works as also given in Ref. [3], the challenge here is to explore whether the pion photoproduction data can be described by only adjusting the bare $\gamma N \rightarrow N^{*}$ vertex $\Gamma_{N^{*}, \gamma N}$ of Eq. (2).

To proceed, we first note that in the study by JLMS [27] it was found that the fit to $\pi N$ elastic scattering data is not sufficient for pinning down precisely the hadronic parameters associated with the unstable particle channels $\pi \Delta, \rho N$, and $\sigma N$. These channels have very large effects in the invariant mass $W \geq$ about $1.65 \mathrm{GeV}$ region where the two-pion production dominates. It is necessary to include the $\pi N \rightarrow \pi \pi N$ data in the fit. This very challenging task is still being pursued at EBAC. For this reason, we will limit our investigation to the $W=1.1 \mathrm{GeV}-1.65 \mathrm{GeV}$ region which covers the energies of the lowlying nucleon resonances in the so called first and second resonance regions. The resulting model is sufficient for investigating the dynamical coupled-channels effects on the $\gamma N \rightarrow \pi N$ cross sections and the $\gamma N \rightarrow N^{*}$ transitions. This is the main focus of this work.

The details of the employed dynamical coupled-channels model have been given in Ref. [3]. In section II we only recall the formulas relevant to the pion photoproduction reactions. The results are presented in section III. Section IV is devoted to discussing the necessary improvements and future developments.

\section{FORMULATION}

In the helicity-LSJ mixed-representation [3] where the initial $\gamma N$ state is specified by its helicities $\lambda_{\gamma}$ and $\lambda_{N}$ and the final $M B$ states by the $(L S) J$ angular momentum variables, the reaction amplitude of $\gamma(\vec{q})+N(-\vec{q}) \rightarrow \pi(\vec{k})+N(-\vec{k})$ at invariant mass $W$ can be written within the MSL formulation as (suppress the isospin quantum numbers)

$$
T_{L S_{N} \pi N, \lambda_{\gamma} \lambda_{N}}^{J}(k, q, W)=t_{L S_{N} \pi N, \lambda_{\gamma} \lambda_{N}}^{J}(k, q, W)+t_{L S_{N} \pi N, \lambda_{\gamma} \lambda_{N}}^{R, J}(k, q, W),
$$

where $S_{N}=1 / 2$ is the nucleon spin, and the non-resonant amplitude is

$$
\begin{aligned}
t_{L S_{N} \pi N, \lambda_{\gamma} \lambda_{N}}^{J}(k, q, E)= & v_{L S_{N} \pi N, \lambda_{\gamma} \lambda_{N}}^{J}(k, q, E)+\sum_{M^{\prime} B^{\prime}} \sum_{L^{\prime} S^{\prime}} \int k^{\prime 2} d k^{\prime} t_{L S_{N} \pi N, L^{\prime} S^{\prime} M^{\prime} B^{\prime}}^{J}\left(k, k^{\prime}, E\right) \\
& \times G_{M^{\prime} B^{\prime}}\left(k^{\prime}, E\right) v_{L^{\prime} S^{\prime} M^{\prime} B^{\prime}, \lambda_{\gamma} \lambda_{N}}^{J}\left(k^{\prime}, q, E\right) .
\end{aligned}
$$


In the above equation, the meson-baryon channels included in the sum are $M^{\prime} B^{\prime}=$ $\pi N, \eta N, \pi \Delta, \rho N, \sigma N$. The matrix elements $v_{L S M B, \lambda_{\gamma} \lambda_{N}}^{J}(k, q, E)$, which describe the $\gamma N \rightarrow$ $M^{\prime} B^{\prime}$ transitions, are calculated from the tree-diagrams, illustrated in Fig. 1, of a set of phenomenological Lagrangians describing the interactions between $\gamma, \pi, \eta, \rho, \omega, \sigma, N$, and $\Delta(1232)$ fields. The details are given explictly in Appendix F of Ref. [3]. We will use the hadronic parameters determined by JLMS [27] to evaluate the meson-baryon propagators $G_{M^{\prime} B^{\prime}}$ and the $\pi N \rightarrow M B$ amplitudes $t_{L S_{N} \pi N, L^{\prime} S^{\prime} M^{\prime} B^{\prime}}^{J}\left(k, k^{\prime}, E\right)$, and to also fix the hadronic vertices of the $\gamma N \rightarrow M B$ amplitudes $v_{L S M B, \lambda_{\gamma} \lambda_{N}}^{J}(k, q, E)$. As discussed in section I, all of the electromagnetic vertices, such as $\gamma N N, \gamma \pi \pi, \gamma \pi \omega$, in $v_{L S M B, \lambda_{\gamma} \lambda_{N}}^{J}(k, q, E)$ are taken from previous works, as also specified in Ref. [3]. Thus the non-resonant amplitude defined by Eq. (5) is completely fixed in the present investigation. Such a consistent dynamical treatment of strong and electromagnetic reaction mechanisms is highly desirable in using the meson production reactions to study the $N^{*}$ structure.

The resonant amplitude in Eq. (4) is

$$
t_{L S_{N} \pi N, \lambda_{\gamma} \lambda_{N}}^{R, J}(k, q, E)=\sum_{N_{i}^{*}, N_{j}^{*}}\left[\bar{\Gamma}_{N_{i}^{*}, L S_{N} \pi N}^{J}(k, W)\right]^{*} D_{i, j}(W) \bar{\Gamma}_{N_{j}^{*}, \lambda_{\gamma} \lambda_{N}}^{J}(q, W),
$$

where the dressed vertex functions are defined as

$$
\begin{aligned}
{\left[\bar{\Gamma}_{N^{*}, L S_{N} \pi N}^{J}(k, W)\right]^{*} } & =\left[\Gamma_{N^{*}, L S_{N} \pi N}^{J}(k)\right]^{*} \\
& +\sum_{M^{\prime} B^{\prime}} \sum_{L^{\prime} S^{\prime}} \int k^{2} d k^{\prime} t_{L S_{N} \pi N, L^{\prime} S^{\prime} M^{\prime} B^{\prime}}^{J}\left(k, k^{\prime}, W\right) G_{M^{\prime} B^{\prime}}\left(k^{\prime}, W\right)\left[\Gamma_{N^{*}, L^{\prime} S^{\prime} M^{\prime} B^{\prime}}^{J}\left(k^{\prime}\right)\right]^{*} \\
\bar{\Gamma}_{N^{*}, \lambda_{\gamma} \lambda_{N}}^{J}(q, W) & =\Gamma_{N^{*}, \lambda_{\gamma} \lambda_{N}}^{J}(q) \\
& +\sum_{M^{\prime} B^{\prime}} \sum_{L^{\prime} S^{\prime}} \int k^{\prime 2} d k^{\prime} \bar{\Gamma}_{N^{*}, L^{\prime} S^{\prime} M^{\prime} B^{\prime}}^{J}\left(k^{\prime}, W\right) G_{M^{\prime} B^{\prime}}\left(k^{\prime}, W\right) v_{L^{\prime} S^{\prime} M^{\prime} B^{\prime}, \lambda_{\gamma} \lambda_{N}}^{J}\left(k^{\prime}, q\right) .(8)
\end{aligned}
$$

The second term of Eq. (8) is due to the mechanism where the non-resonant electromagnetic meson production takes place before the dressed $N^{*}$ states are formed. The contribution due to the $\pi N$ intermediate state is illustrated in Fig. 2. Similar to what was defined in Ref. [11, 19], we call this contribution the meson cloud effect to define precisely what will be presented in section III.

The $N^{*}$ propagator in Eq. (6) is defined by

$$
\left[D(W)^{-1}\right]_{i, j}=\left(W-M_{N_{i}^{*}}^{0}\right) \delta_{i, j}-\bar{\Sigma}_{i, j}(W)
$$

where $M_{N_{i}^{*}}^{0}$ is the bare mass of the $N_{i}^{*}$ state, and the self-energies are

$$
\bar{\Sigma}_{i, j}(W)=\sum_{M B} \sum_{L S} \int d k k^{2} \Gamma_{N_{i}^{*}, L S M B}^{J}(k) G_{M B}(k, W)\left[\bar{\Gamma}_{N_{j}^{*}, L S M B}^{J}(k, W)\right]^{*} .
$$

Since the dressed vertex $\bar{\Gamma}_{N^{*}, L S M B}^{J}(k, W)$ of $N^{*} \rightarrow M B$ in Eqs. (7)-(8) and the bare masses $M_{N^{*}}^{0}$ and the mass shifts $\bar{\Sigma}_{i, j}$ in Eq. (9) have been determined by JLMS [27], the only unknown quantities in the resonant amplitude Eq. (6) are the bare $\gamma N \rightarrow N^{*}$ vertex functions $\Gamma_{N^{*}, \lambda_{\gamma} \lambda_{N}}^{J}(q)$ in Eq. (8). We parameterize these functions as

$$
\Gamma_{N^{*}, \lambda_{\gamma} \lambda_{N}}^{J}(q)=\frac{1}{(2 \pi)^{3 / 2}} \sqrt{\frac{m_{N}}{E_{N}(q)}} \frac{1}{\sqrt{2 q}}\left[\sqrt{2 q_{R}} A_{\lambda}^{J}\right] \delta_{\lambda,\left(\lambda_{\gamma}-\lambda_{N}\right)},
$$




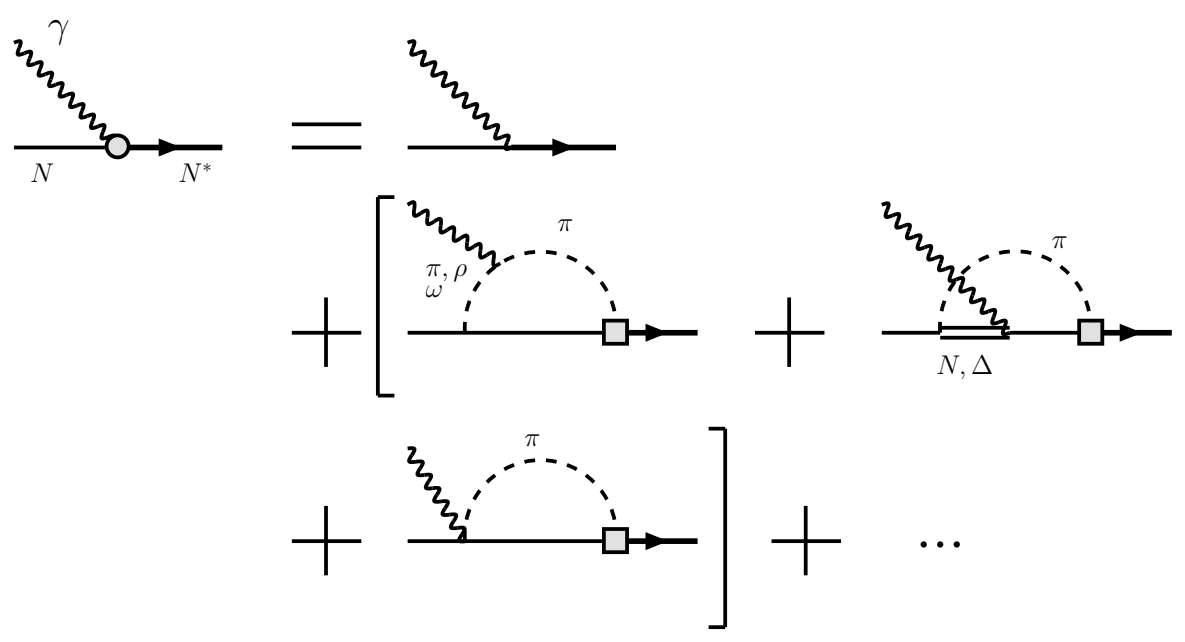

FIG. 2: Graphical illustration of the contribution to the $\pi N$ intermediate state to the dressed $\gamma N \rightarrow N^{*}$ vertex defined by Eq. (8).

where $q_{R}$ is defined by the $N^{*}$ mass $M_{N^{*}}=q_{R}+E_{N}\left(q_{R}\right)$. For later discussions, we also cast the dressed vertex Eq. (8) into the form of Eq. (11) with a dressed helicity amplitude

$$
\bar{A}_{\lambda}^{J}(q)=A_{\lambda}^{J}+A_{\lambda}^{m c, J}(q)
$$

where $A_{\lambda}^{m c, J}(q)$ is due to the meson cloud effects defined by the second term of Eq. (8). The dressed helicity amplitude $\bar{A}_{\lambda}^{J}$ is related to the partial width of the electromagnetic decay of a $N^{*}$ resonance defined by

$$
[\text { partial decay width }]=\frac{q_{R}^{2}}{4 \pi} \frac{m_{N}}{M_{N^{*}}} \frac{8}{2 J+1}\left[\left|\bar{A}_{3 / 2}^{J}\left(q_{R}\right)\right|^{2}+\left|\bar{A}_{1 / 2}^{J}\left(q_{R}\right)\right|^{2}\right] .
$$

Eqs. (11)-(13) and Eq. (8) fix the relation between the bare helicity amplitude $A_{\lambda}^{J}$ of Eq. (8) and the helicity amplitudes $\bar{A}_{\lambda}^{J}$ listed by the Particle Data Group (PDG).

\section{RESULTS}

With the specifications given in section II, our task is to perform $\chi^{2}$-fits to the available data of $\gamma N \rightarrow \pi N$ reactions up to $W=1.65 \mathrm{GeV}$ by adjusting the bare helicity amplitudes $A_{\lambda}^{J}$ of the bare $N^{*}$ states included in the JLMS fit [27] to the $\pi N$ scattering data. The $\chi^{2}$-fits are performed by using MINUIT. In this first and second resonance regions, we find that the fits to the data are mainly sensitive to the bare helicity amplitudes listed in Table I. Other bare helicity amplitudes are set to zero in the calculations. The quality of the resulting fit can be seen in Figs. 3, 4, 5 and 6. The solid curves in Figs. 3 and 4 are the calculated differential cross sections $(d \sigma / d \Omega)$ for $\gamma p \rightarrow \pi^{0} p$ and $\gamma p \rightarrow \pi^{+} n$, respectively, compared with the corresponding experimental data. Similarly solid curves in Figs. 5 and 6 correspond to the obtained photon asymmetry $\left(\Sigma_{\gamma}\right)$ compared to the experimental data for the reactions $\gamma p \rightarrow \pi^{0} p$ and $\gamma p \rightarrow \pi^{+} n$, respectively. We see that the model can give an overall good description of the considered data, while significant discrepancies with the data remain. 


\begin{tabular}{c|cc}
\hline \hline Bare $N^{*}$ & $A_{1 / 2}\left[10^{-3} \mathrm{GeV}^{-1 / 2}\right]$ & $A_{3 / 2}\left[10^{-3} \mathrm{GeV}^{-1 / 2}\right]$ \\
\hline$S_{11}-1$ & 98 & - \\
\hline$S_{11}-2$ & 11 & - \\
\hline$S_{31}-1$ & 197 & - \\
\hline$P_{11}-1$ & 33 & - \\
\hline$P_{13}-1$ & -124 & -94 \\
\hline$P_{33}-1$ & -112 & -204 \\
\hline$D_{13}-1$ & 32 & -30 \\
\hline$D_{15}-1$ & 11 & 3.2 \\
\hline$D_{33}-1$ & 84 & -46 \\
\hline$F_{15}-1$ & -107 & -73 \\
\hline \hline
\end{tabular}

TABLE I: The bare $\gamma N \rightarrow N^{*}$ helicity amplitudes determined from $\chi^{2}$-fits to the $\gamma N \rightarrow \pi N$ data shown in Figs. 3-6.

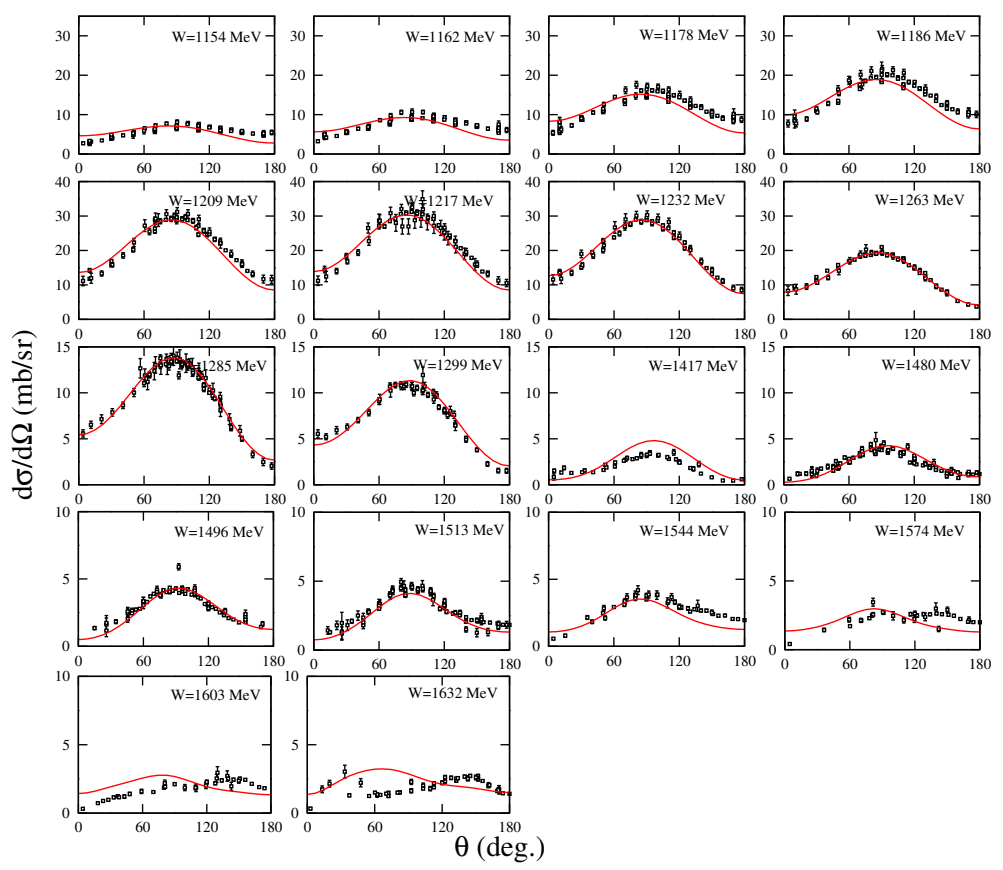

FIG. 3: Differential cross section for $\gamma p \rightarrow \pi^{0} p$ compared to experimental data obtained from Ref. [28].

We emphasize here that the determined bare helicity amplitudes listed in Table I are not directly the properties associated with the nucleon resonances. They are simply the properties of the excited nucleon states in the absence of coupling to the reaction channels. We need to identify the resonant positions from the partial-wave amplitudes predicted by our model. The dressed helicity amplitudes $\bar{A}_{\lambda}^{J}$ calculated at those resonance positions according to Eq. (8) can then be compared to the partial decay widths from the measurements, as seen in Eq. (13). This is being pursued by developing [29] an analytic continuation method to evaluate the reaction amplitudes in the complex energy plane following the dynamical coupled-channels equations of the MSL model [3]. 


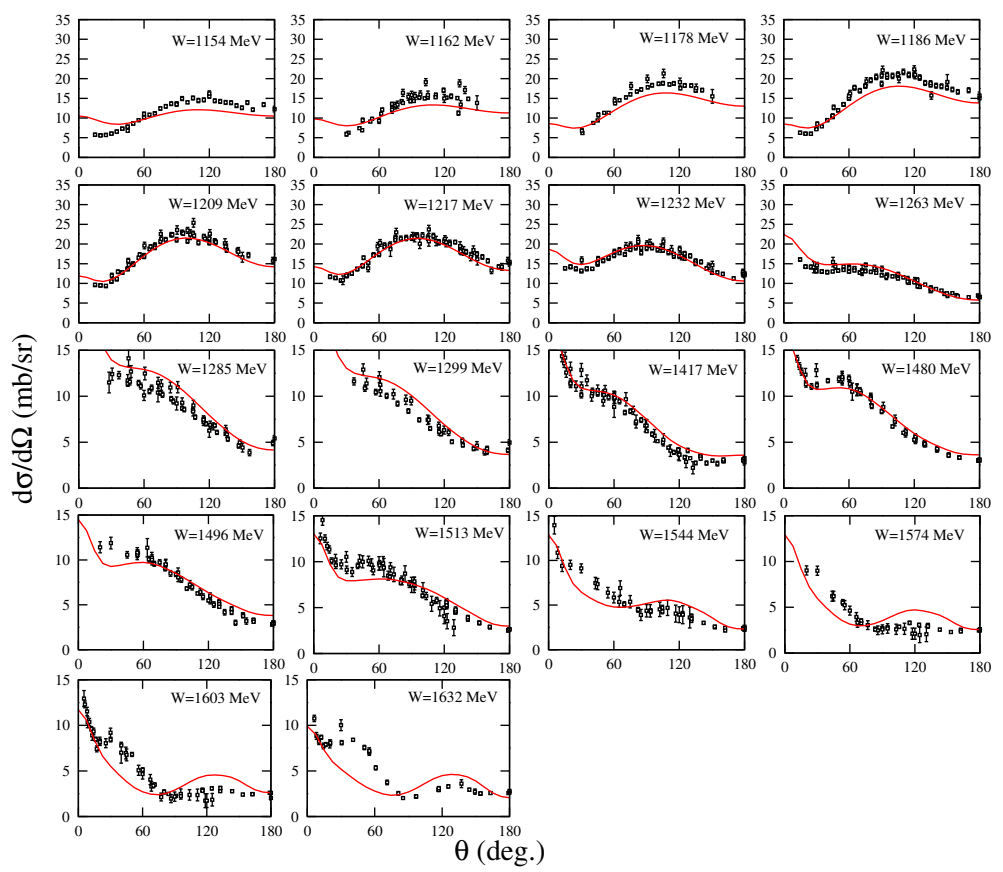

FIG. 4: Differential cross section for $\gamma p \rightarrow \pi^{+} n$ compared to experimental data obtained from Ref. [28].

We now turn to investigating the coupled-channels effects. In Fig. 7, we see that the calculated total cross sections (solid curves) are in good agreement with the data. The dashed curves are obtained when the channels $\eta N, \pi \Delta, \rho N$, and $\sigma N$ are turned off in the loop integrations in the non-resonant amplitude defined by Eq. (5) and the dressed $\gamma N \rightarrow N^{*}$ vertex defined by Eq. (8). Clearly, the coupled-channels effects $\gamma N \rightarrow \eta N, \pi \Delta, \rho N, \sigma N$ $\rightarrow \pi N$ can change the cross sections by about $10-20 \%$ in the $\Delta$ (1232) region and as much as $50 \%$ in the $W>1400 \mathrm{MeV}$ second resonance region. The corresponding coupled-channels effects on the differential cross sections are shown in Fig. 8. Comparing the solid and dashed curves, we see that the coupled-channels effects can change the magnitudes and shapes of $d \sigma / d \Omega$, in particular at higher energies.

As discussed in the introduction, an essential feature of a dynamical approach is to solve integral equations Eqs. (1)-(2) which involve off-shell amplitudes. The off-shell effects are due to the reaction mechanisms at short distances which are not treated explicitly in the technically much simpler K-matrix coupled-channels models [4-6, 20-22]. If the off-shell effects are neglected in the $\pi N$-loop only calculations, we then bring the dashed curves in Figs.7 and 8 to the dotted curves. Clearly, the off-shell effects are very significant, as was also revealed in the coupled-channels calculations [18] of $K Y$ photoproduction.

To disentangle the structure of nucleon resonances, it is important to investigate the meson cloud effect on the $\gamma N \rightarrow N^{*}$, as defined by the second term of Eq. (8). It can have contributions from the loop integrations over the $\pi N, \eta N, \pi \Delta, \rho N, \sigma N$ intermediate states in this calculation. The contribution from the $\pi N$ loop is that illustrated in Fig. 2 . If the electromagnetic form factors associated with the non-resonant interaction $v_{M B, \gamma N}$ are taken from previous works, we can predict the $Q^{2}$-dependence of the meson cloud effect term $A_{\lambda}^{m c, J}$ of Eq. (12).

We first investigate the $\gamma N \rightarrow \Delta$ (1232) transition. The resonance position of $\Delta(1232)$ is 


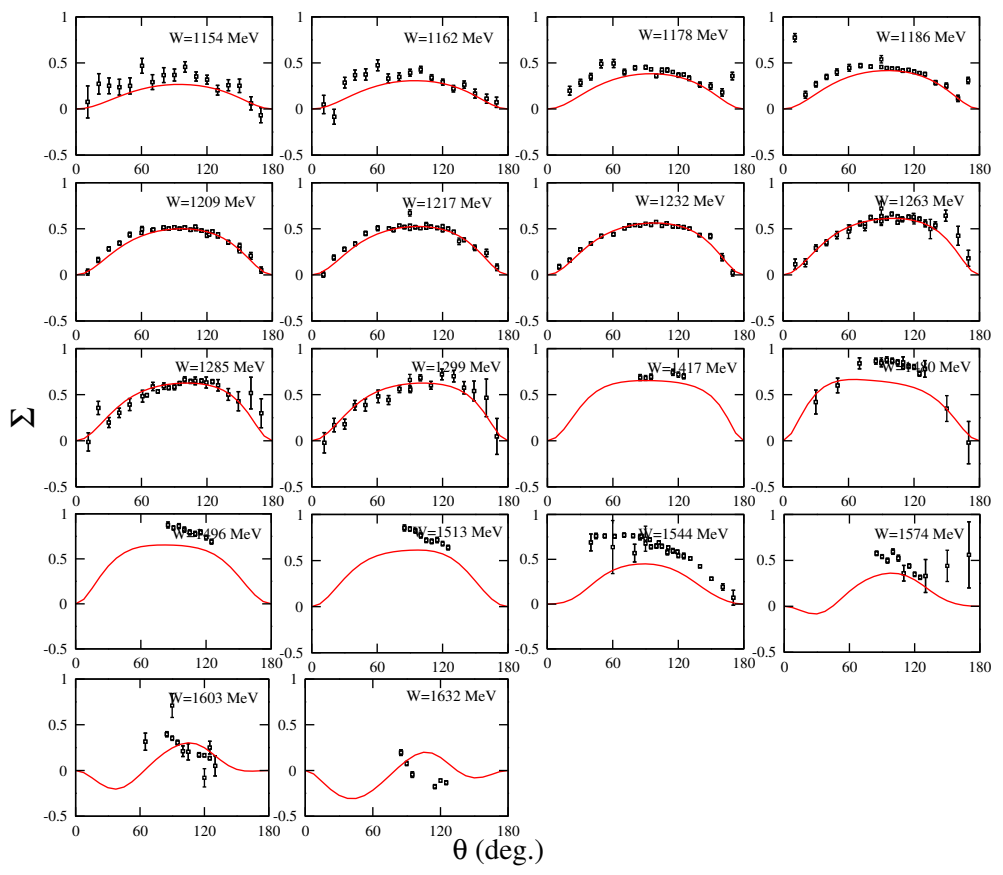

FIG. 5: Photon asymmetry, $\Sigma_{\gamma}$, for $\gamma p \rightarrow \pi^{0} p$ compared to experimental data obtained from Ref. [28].

well defined and reproduced in our calculations as can be seen in the good description of the cross section data near $W=1232 \mathrm{MeV}$ in Figs. 3-7. For this isolated resonance, we can follow the procedures detailed in Ref. [11] to calculate the $\gamma N \rightarrow \Delta$ (1232) magnetic form factor $G_{M}^{*}\left(Q^{2}\right)$ from the imaginary part of the multipole amplitude $M_{1^{+}}^{I=3 / 2}$ of $\gamma^{*} N \rightarrow \pi N$ reactions at $W=1232 \mathrm{MeV}$. Our results are shown in Fig. 9. The dashed curve corresponds to the full meson cloud contribution from this calculation. The dotted curve is obtained by keeping only the $\pi$-loop in the calculation. Clearly the difference between the dashed and dotted curves is due to the coupled-channels effects $\gamma N \rightarrow \eta N, \pi \Delta, \rho N, \sigma N \rightarrow \Delta$ (1232). Here we note that the pion-loop only result (dotted curve) is very close to the meson cloud effect predicted by the Sato-Lee Model[11]. The solid curve following the data is just for guiding the eyes. The difference between the solid curve and the dashed curve provide information about the $Q^{2}$-dependence of the bare $\gamma N \rightarrow \Delta$ (1232) form factor which can be used as the starting point of our dynamical coupled-channels analysis of pion electroproduction.

For the meson cloud effects on the other $\gamma N \rightarrow N^{*}$ vertex, we calculate the second term of Eq. (8) to get $A_{\lambda}^{m c, J}$ by using the normalization defined by Eqs. (11)-(12). In Fig. 10, we show the predicted magnitudes of $\left|A_{\lambda}^{m c, J}\right|$ evaluated at $W=1535 \mathrm{MeV}$ for $S_{11}, W=1440$ $\mathrm{MeV}$ for $P_{11}, W=1520 \mathrm{MeV}$ for $D_{13}, W=1625 \mathrm{MeV}$ for $D_{15}$, and $W=1620 \mathrm{MeV}$ for $S_{31}$. The solid dots at $Q^{2}=0$ are the determined bare helicity amplitudes. The solid curves are from the full calculations and the dashed curves are from only keeping the $\pi N$ loop in Eq. (8). We see that the meson cloud contributions and the coupled-channels effects affect mainly the low $Q^{2}$ region.

Here we note that the results presented in Fig. 10 are around the resonance positions listed by PDG, not from the resonance pole positions which will be determined [29] using an analytical continuation [29]. Thus the results presented here are only for giving some qualitative estimate of the meson cloud effects on $\gamma N \rightarrow N^{*}$ excitation. More accurate predictions 


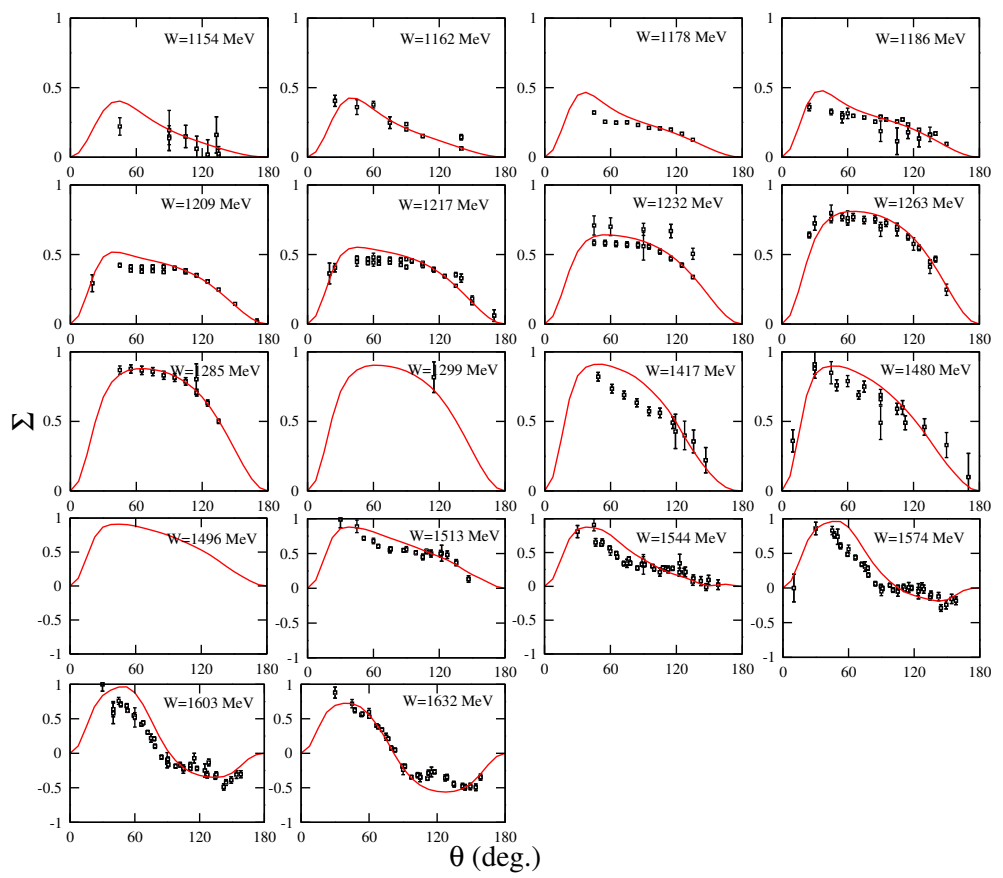

FIG. 6: Photon asymmetry, $\Sigma_{\gamma}$, for $\gamma p \rightarrow \pi^{+} n$ compared to experimental data obtained from Ref. [28].
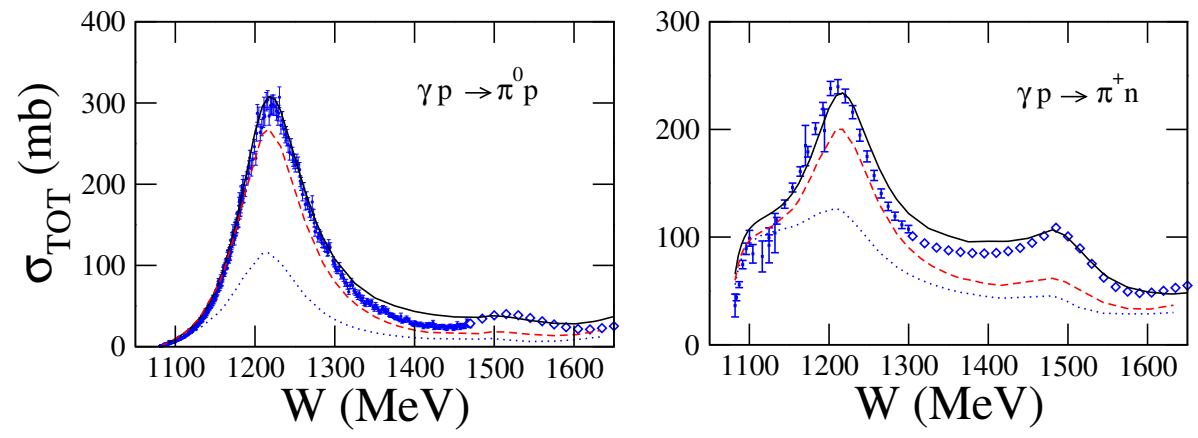

FIG. 7: Total cross sections. The dashed curves are obtained from turning off all $M B$ channels except the $\pi N$ channel in the the loop integrations in the non-resonant amplitude defined by Eq. (5) and the dressed $\gamma N \rightarrow N^{*}$ vertex defined by Eq. (8). The dotted curve is obtained by neglecting the off shell effects in the $\pi N$ only calculation. Experimental data obtained using Ref. [28]. The diamonds correspond to the SAID solution [20].

will be published in our subsequent analysis [29] of the data of pion electroproduction.

\section{SUMMARY AND FUTURE DEVELOPMENTS}

We have applied the dynamical coupled-channels model of Ref. [3] to investigate the pion photoproduction reactions in the first and second nucleon resonance region. With the hadronic parameters of the model determined in a recent study [27] of $\pi N$ scattering data 


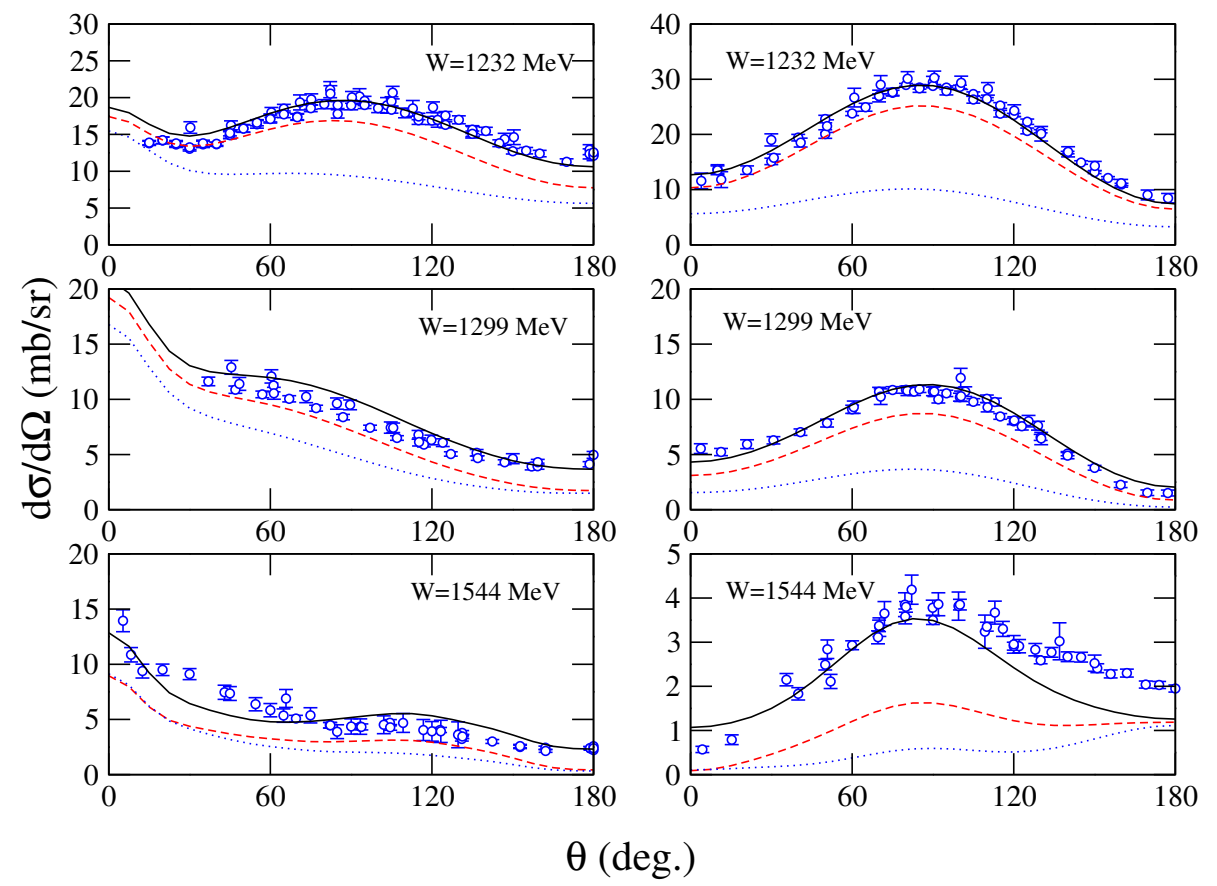

FIG. 8: coupled-channels effects on $d \sigma / d \Omega$ at $\mathrm{W}=1.23,1.30,1.54 \mathrm{GeV}$. The dashed curves are obtained from turning off all $M B$ channels except the $\pi N$ channel in the the loop integrations in the non-resonant amplitude defined by Eq. (5) and the dressed $\gamma N \rightarrow N^{*}$ vertex defined by Eq. (8). The dotted curve is obtained by neglecting the off shell effects in the $\pi N$ only calculation. Right-hand side $: \gamma p \rightarrow \pi^{0} p$, left-hand side $: \gamma p \rightarrow \pi^{+} n$

and the non-resonant electromagnetic couplings taken from the previous works, we show that the available data of differential cross sections and photon asymmetries of $\gamma N \rightarrow \pi N$ up to $W=1.65 \mathrm{GeV}$ can be described to a very large extent. The only free parameters in the $\chi^{2}$-fit to the data are the bare $\gamma N \rightarrow N^{*}$ helicity amplitudes. It is found that the coupledchannels effects can have about 10 - $20 \%$ effects in the $\Delta$ (1232) resonance region, and can drastically change the magnitudes and shapes of the cross sections in the second resonance region. We also demonstrate the importance of the off-shell effects in a dynamical approach. The meson cloud contributions to the $\gamma N \rightarrow N^{*}$ form factors have been predicted. For all cases, they are mainly in the low $Q^{2}$ region. For the magnetic M1 $\gamma N \rightarrow \Delta$ (1232) form factor, the results are close to those of the Sato-Lee Model. The coupled-channels effects on the meson cloud contributions are also found to be mainly in the low $Q^{2}$ region.

The results presented here can be the starting point for analyzing the pion electroproduction data in the first and second resonance region. It will be interesting to see the bare $\gamma N \rightarrow \Delta$ (1232) form factor which can be estimated from the differences between the solid and dashed curves in Fig. 9 can be verified in the analysis of the data at $W=1232 \mathrm{MeV}$ and $Q^{2} \leq 6(\mathrm{GeV} / \mathrm{c})^{2}$. For the data in the second resonance region, our task is to extract the bare helicity amplitudes of $\gamma N \rightarrow N^{*}$ at each $Q^{2}$, as was done in this work at the photon point $Q^{2}=0$. Of course the data to be fitted are much more extensive and we need to also determine the longitudinal components of the $\gamma N \rightarrow N^{*}$ vertex. This is being pursued [30] 


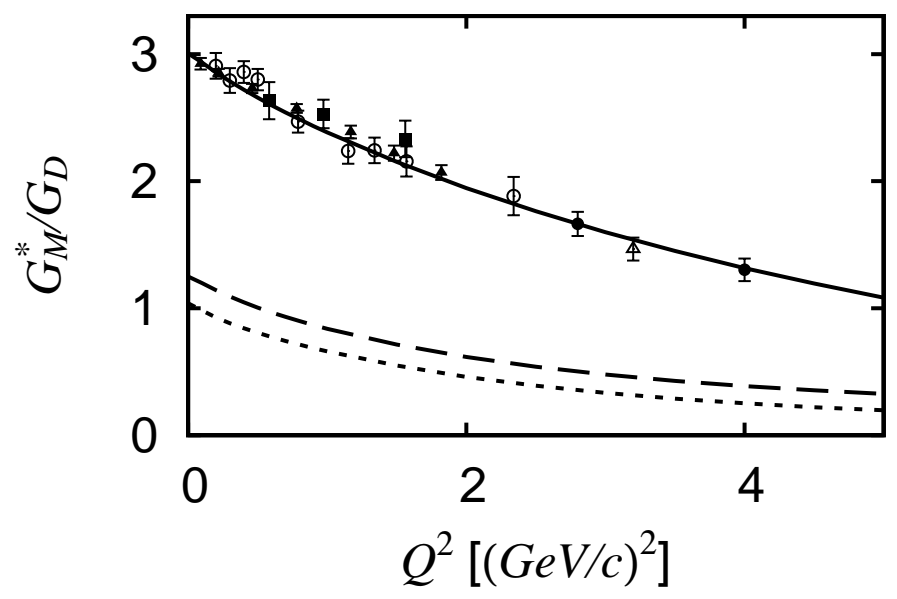

FIG. 9: The predicted $Q^{2}$-dependence of meson cloud contributions to the magnetic M1 form factor $G_{M}^{*}$ defined in Ref. [11] and $G_{D}\left(Q^{2}\right)=1 /\left[1+Q^{2} /\left(0.71(G e V / c)^{2}\right)\right]^{2}$. The dashed curve in the lower part of the figure is the predicted full meson cloud contribution. The dotted curve is obtained after turning off the coupled-channels effects due to $\pi \Delta$ and $\rho N$ channels in the loop integrations of Eq. (8). The data are compiled in Ref. [19].

at EBAC.

The most unsatisfactory part of this work is the uncertainties in determining the bare $\gamma N \rightarrow N^{*}$ helicity amplitudes. As seen in Table I, there are 16 helicity amplitudes adjusted in our $\chi^{2}$-fits to the data. Obviously, it is possible that there could exist other solutions to the minimization problem if more experimental data, in particular polarization data is included in the fit. Within the MSL formulation [3], these bare parameters are related to hadron structure calculations in the absence of the couplings with reaction channels. A more fruitful approach is to take the helicity amplitudes predicted from such hadron structure calculations as the starting values in our $\chi^{2}$-fit to avoid unphysical solutions. The resulting parameters could then be used to examine that hadron structure calculation. However more theoretical analysis is needed to know precisely what kind of structure calculations are consistent with the MSL formulation and can be used for this purpose. Since it was found [19] that the extracted bare $\gamma N \rightarrow \Delta$ (1232) magnetic M1 form factor is fairly consistent with the prediction of constituent quark models, one possibility is to use the relativistic constituent quark model [31].

To improve the agreement with the data, one necessary next step is to improve the hadronic parameters of the model. These parameters, fixed at the values from the fit to $\pi N$ scattering data, must be improved by performing a combined analysis of both the $\pi N$ elastic scattering and $\pi N \rightarrow \pi \pi N$ data. We expect that the parameters associated with the unstable particle channels, $\pi \Delta, \rho N$, and $\sigma N$, will be refined most significantly. Thus the predicted coupled-channels effects on $\gamma N \rightarrow \pi N$ cross sections, as shown in Fig. 8 will be changed such that the fits to the data shown in Figs. 3-6, in particular in the high $W$ region, can be improved. Furthermore, this is also needed to extend our investigation to the third 

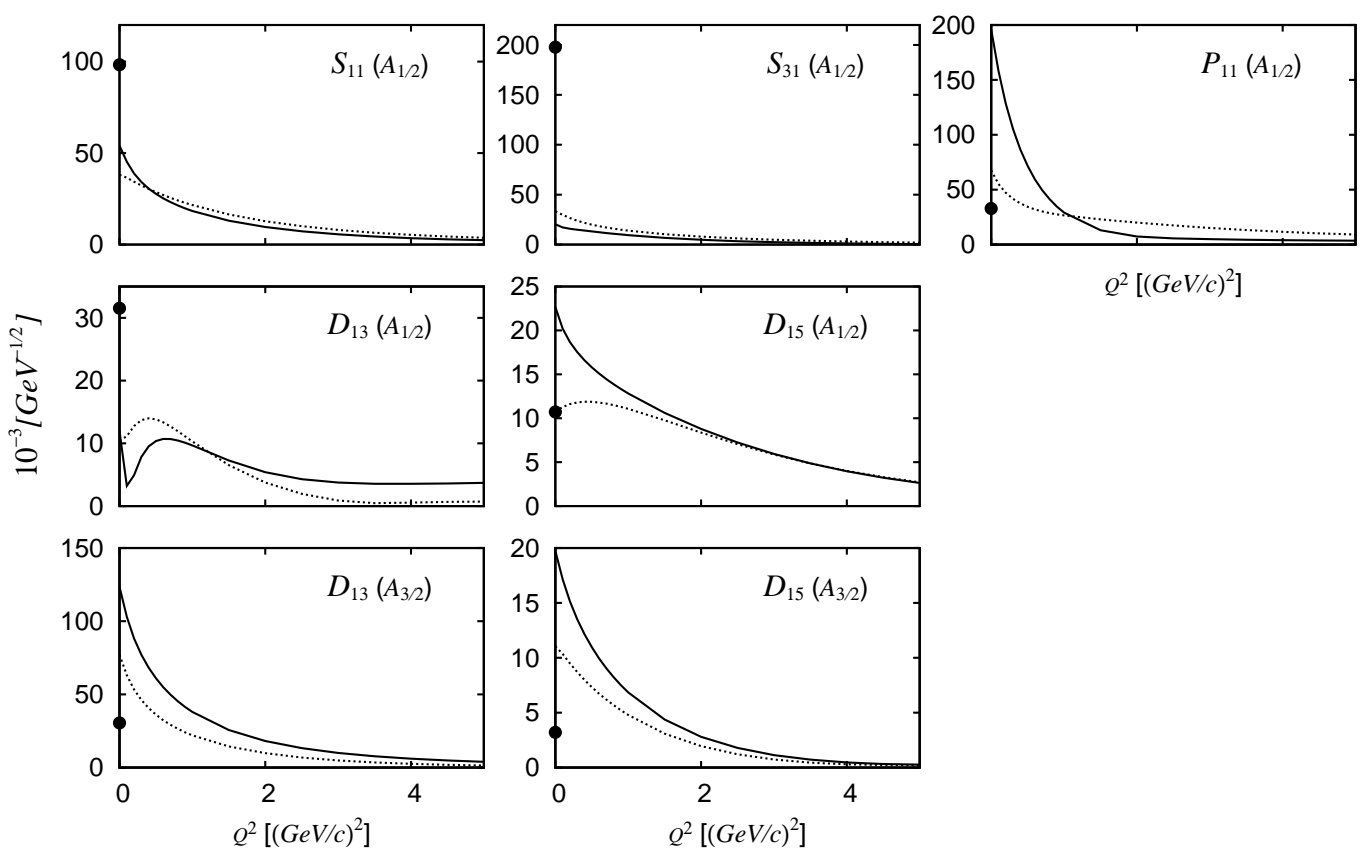

FIG. 10: The predicted $Q^{2}$-dependence of meson cloud contributions to the helicity amplitudes $\bar{A}_{\lambda}^{J}$. The dotted curves are from keeping only the $\pi$-loop in Eq. (8). The black dot corresponds to the absolute value of the bare helicities listed in Table. I.

resonance region where the two pion production dominates and the coupled-channels effects through these unstable particle channels are expected to be very large.

Finally, we would like to address the questions concerning how the results from JLMS and this investigation can be used to extract the positions and widths of nucleon resonances. The resonances positions are the poles of the reaction amplitudes in the complex energy plane. The residues of these poles can then be related to the partial decay widths. If these poles are identical to the zeros of the $N^{*}$ propagator $D_{i, j}(E)$ Eq. $(9)$, we then have the most desirable interpretation that the nucleon resonance is due to the coupling of the bare $N^{*}$ with the reaction channels. The dressed vertex functions $\Gamma_{N^{*}, \gamma N}$, defined by Eq. (8), evaluated at these poles can be used to predict the dressed helicity amplitudes $\bar{A}_{\lambda}$ for calculating the partial decay widths using Eq. (13). On the other hand, the poles could be from the nonresonant amplitude such as the term $t_{L S_{N} \pi N, \lambda_{\gamma} \lambda_{N}}^{J}$ of Eq. (4). Then the identified resonances have nothing to do with the bare $N^{*}$ states and are simply due to the attractive mesonbaryon interactions. Extraction of this resonance information requires developing numerical methods for solving the dynamical coupled-channels equations on the complex energy plane. Our effort in this direction will be published elsewhere [29].

\section{Acknowledgments}

We would like to thank Dick Arndt for useful discussions. B.J-D acknowledges the support of the Japanese Society for the Promotion of Science (JSPS), grant number: PE 07021. B.J- 
D. thanks the nuclear theory group at Osaka University for their warm hospitality. This work is supported by the U.S. Department of Energy, Office of Nuclear Physics Division, under contract No. DE-AC02-06CH11357, and Contract No. DE-AC05-060R23177 under which Jefferson Science Associates operates Jefferson Lab, and by the Japan Society for the Promotion of Science, Grant-in-Aid for Scientific Research(c) 15540275. This work is also partially supported by Grant No. FIS2005-03142 from MEC (Spain) and FEDER and European Hadron Physics Project RII3-CT-2004-506078. 
[1] V. Burkert and T.-S. H. Lee, Int. J. of Mod. Phys. E13, 1035 (2004).

[2] T.-S. H. Lee and L.C. Smith, J. Phys. G 34, 1 (2007).

[3] A. Matsuyama, T. Sato, and T.-S. H. Lee Phys. Rept. 439, 193 (2007)

[4] Feuster and U. Mosel, Phys. Rev. C 58, 457 (1998); Phys. Rev. C 59, 460-491 (1999); V. Shklyar, H. Lenske, U. Mosel, G. Penner, Phys. Rev. C 71, 055206 (2005).

[5] A. Usov and O. Scholten, Phys. Rev. C 72, 025205 (2005).

[6] A.V. Sarantsev, V.A. Nikonov, A. V. Anisovich, E. Klempt, and U. Thoma, Eur. Phys. J. A 25, $441(2005)$.

[7] G. Y. Chen, S. S. Kamalov, S. N. Yang, D. Drechsel and L. Tiator, Phys. Rev. C 76, 035206 (2007) [arXiv:nucl-th/0703096].

[8] S.N. Yang, J. of Phys. G11, L205 (1985)

[9] S. Nozawa, B. Blankleider and T.-S. H. Lee, Nucl. Phys. A 513, 459 (1990).

[10] F. Gross and Y. Surya, Phys. Rev. C 47, 703 (1993).

[11] T. Sato and T.-S. H. Lee, Phys. Rev. C 54, 2660 (1996); Phys. Rev. C 63, 055201 (2001).

[12] S. S. Kamalov and S.N. Yang, Phys. Rev. Lett. 83, 4494 (1999).

[13] O. Krehl, C. Hanhart, S. Krewald, and J. Speth, Phys. Rev. C 60, 055206 (1999); C 62, 025207 (2000).

[14] A.D. Lahiff and I.R. Afnan, Phys. Rev. C 66, 044001 (2002).

[15] M.G. Fuda and H. Alharbi, Phys. Rev. C 68, 064002 (2003).

[16] V. Pascalutsa and J. Tjon, Phys. Rev. C 61, 054003 (2000).

[17] W. T. Chiang, F. Tabakin, T.-S. H. Lee and B. Saghai, Phys. Lett. B 517, 101 (2001)

[18] B. Julia-Diaz, B. Saghai, T.-S. H. Lee and F. Tabakin, Phys. Rev. C 73, 055204 (2006).

[19] B. Julia-Diaz, T.-S. H Lee, T. Sato and L. C. Smith, Phys. Rev. C 75, 015205 (2007).

[20] R.A. Arndt, I.I. Strakovsky, R.L. Workman, Phys. Rev. C 53, 430 (1996); Int. J. Mod. Phys. A18, 449 (2003), Phys. Rev. C 66, 055213 (2002).

[21] D. Drechsel, O. Hanstein, S.S. Kamalov, and L. Tiator, Nucl. Phys. A645, 145 (1999); S.S. Kamalov, S. N. Yang, D. Drechsel, O. Hanstein, and L. Tiator, Phys, Rev, C 64, 032201(R) (2001).

[22] I. G. Aznauryan, Phys. Rev. C 68, 065204 (2003).

[23] S. Capstick and W. Roberts, Phys. Rev. D 49, 4570 (1994).

[24] P. Maris and C. D. Roberts, Int. J. Mod. Phys. E 12, 297 (2003).

[25] C. Alexandrou, G. Koutsou, T. Leontiou, J. W. Negele and A. Tsapalis, Phys. Rev. D 76, 094511 (2007).

[26] D. Richards, arXiv:0711.2048 [nucl-th].

[27] B. Julia-Diaz, T.-S. H. Lee, A. Matsuyama, T. Sato, Phys. Rev. C 76, 065201 (2007).

[28] CNS Data Analysis Center, George Washington University, http://gwdac.phys.gwu.edu.

[29] N. Suzuki, T. Sato, T.-S. H. Lee, MENU2007 proceedings, to be published.

[30] B. Julia-Diaz, T.-S. H. Lee, A. Matsuyama, T. Sato, L.C. Smith, in preparation.

[31] B. Julia-Diaz, D. O. Riska and F. Coester, Phys. Rev. C 69, 035212 (2004), [Erratum-ibid. C 75, $069902(2007)]$. 